\title{
EL ARTE CONTEMPORÁNEO: UN ARTE DE RAZONES
}

\author{
Contemporary art: an art of raisons \\ Sebastián Díaz \\ Universidad Nacional de Tucumán, Tucumán, Argentina \\ sebastiandiazfi@gmail.com \\ María Gallo Ugarte \\ Universidad Nacional de Tucumán, Tucumán, Argentina \\ airamag@yahoo.com.ar
}

\begin{abstract}
Resumen
La historia del arte presupuso distintas formas de comprensión. Con el arte moderno y aún más con el arte contemporáneo se profundizó la grieta en la relación del arte con sus razones, una grieta cada vez mayor entre percepción y juicio estético; por ello es que las producciones que se ubican en lo que se denomina 'arte contemporáneo' exigen que se atienda no sólo a los estímulos perceptuales que nos puede provocar la obra sino, sobre todo, a sus razones. El objetivo del trabajo es hacer un análisis de la tensión resultante entre los defensores de la estética tradicional y quienes producen (artistas), exhiben (galeristas, curadores, etc.), consumen (espectadores) o defienden (teóricos del arte, críticos, estetas) obras que se ubican fuera de la lógica desplegada por aquella estética. El presente análisis lo llevaremos a cabo a partir de los aportes del segundo período de la filosofía de Wittgenstein, nociones como las de juego de lenguaje, formas de vida, apreciación, etc., son algunas de las herramientas para pasar la discusión por el tamiz. Esperamos con ello contribuir a enriquecer algunas discusiones que se desprenden en torno a la problemática del arte contemporáneo. La hipótesis es que la estética actual puede valerse de este renovado aparato conceptual desplegado por la filosofía wittgensteniana a partir de la década del 30'.
\end{abstract}

Palabras clave: juicio estético, criterios, juego de lenguaje, arte contemporáneo, apreciación.

\begin{abstract}
History of art presupposed different ways of comprehension. With modern art and even more with contemporary art, the rift between art and its reasons was deepened, a growing rift between perception and aesthetic judgment; that is why the productions that we identify as contemporary art demand that we pay attention not only to the perceptual stimuli that the work can provide us with but also to its raisons above all. The aim of the work is to make an analysis of the resulting tension between the defenders of traditional aesthetics and those who produce (artists), exhibit (gallery owners, curators), consume (spectators), or defend (art theorists, reviewers) works that are located outside the logic displayed by that aesthetic. The present analysis will be carried out from the contributions we are going to use of the second period of Wittgenstein's philosophy, notions such as language-games, life forms, appreciation, etc., are some of the tools to pass the discussion through the sieve. We hope to contribute to enrich some discussions that arise around the problem of contemporary art. The hypothesis is that current aesthetics are set up to a large extent based on this new conceptual device displayed by Wittgenstein's philosophy from the 30's.
\end{abstract}

Keywords: aesthetic judgment, criteria, language-games, contemporary art, appreciation.

Fecha de Recepción: 30/06/2018 - Fecha de Aceptación: 26/12/2018 
Las razones del arte que casi siempre derivan de las sinrazones del mundo, las razones de lo que nunca ha sido dicho o simbolizado. Hacer arte es quebrar el orden de los significados para hacer surgir un nuevo fragmento de sentido, algo que desordena lo viejo.

(Gerard Vilar, Las razones del arte)

\section{Introducción}

En el presente trabajo nos proponemos abordar el asunto de los juicios en el terreno de la estética entendida como disciplina filosófica: ¿dónde encuentran su asidero, su "legitimidad" las afirmaciones de valor? ¿En este terreno vale acaso lo mismo toda apreciación? En otras palabras, la pregunta que servirá de eje podría enunciarse del siguiente modo: ¿cómo legitimamos un juicio estético por sobre otro?, ¿cómo hacerlo cuando aparentemente en el arte contemporáneo lo que se celebra pareciera ser justamente la ausencia de todo criterio extra-subjetivo? Para adentrarnos en esta temática nos valdremos de algunas herramientas que nos proporciona la filosofía del lenguaje desarrollada por el llamado "segundo Wittgenstein", sobre todo a partir de Las Investigaciones filosóficas. Entramos, así, a la problemática de la argumentación en el terreno de la estética, es decir, las respuestas posibles a la pregunta sobre la fundamentación de los juicios estéticos. Además, haremos uso de artículos relevantes acerca de la relación entre la filosofía del segundo Wittgenstein y la estética, así como de otros escritos suyos como las Lecciones y conversaciones sobre estética, psicología y creencia religiosa, Comentarios a la Rama Dorada de Frazer, Los cuadernos azul y marrón, etc.

Valiéndonos del pasaje de una teoría del lenguaje formalista a una anclada en los juegos de lenguaje y las formas de vida, intentaremos dar posibles orientaciones ante estos interrogantes, sin pretender llegar a una respuesta definitiva. Entendemos que toda respuesta es susceptible de crítica, y ésta no será la excepción.

\section{El nuevo aparato conceptual wittgensteiniano}

El filósofo mexicano Alejandro Tomasini Bassols en su artículo La superioridad del método de los juegos de lenguaje y las formas de vida (2012) postula dos grandes concepciones del lenguaje que emergen de la filosofía de Wittgenstein. La concepción tradicional del lenguaje, que alcanza su formulación más acabada en el Tractatus Logico-Philosophicus (Wittgenstein 1979) ${ }^{1}$, sostiene una filosofía del lenguaje formalista y referencialista. Ese formalismo radica en la identidad

\footnotetext{
${ }^{1}$ En adelante citaremos el Tractatus Logico-Philosophicus y las Investigaciones filosóficas indicando no el número de página sino el número del parágrafo, precedido del siguiente símbolo §.
} 
estructural, lógica, que supone debe existir entre lenguaje y realidad. Ciertamente, la teoría pictórica intenta dar cuenta de la representación en un sentido general, donde se incluye a la representación lingüística y se toma como esquema ejemplar el de un tipo particular de figuración: el de las proposiciones. Sin entrar en detalles, aquí la propuesta wittgensteiniana sostiene que la función lógica (diríamos, básica) del lenguaje es retratar, figurar (figura: Bild) hechos posibles (cuyo conjunto conforma la realidad, Wirklichkeit). Debe entonces haber algo idéntico entre la figura y lo figurado para que pueda haber figuración: un hecho para poder ser figura, debe tener algo en común con lo figurado (1979 §2.16). Eso en común es la forma pictórica.

La consecuencia de este "isomorfismo estructural" es una suerte de universalismo semántico en el que todo lo que pueda ser dicho es una determinada combinación que comparte una estructura con la combinación que retrata. De aquí que Wittgenstein identifique lenguaje significativo con lenguaje científico: el verdadero método de la filosofía sería propiamente éste: no decir nada, sino aquello que se puede decir; es decir, las proposiciones de la ciencia natural (1979 $\S 6.53)$.

Esto es importante porque permite visibilizar los límites precisos que para este período de la filosofía de Wittgenstein tiene el lenguaje con sentido. Su teoría pictórica, al plantear la imposibilidad de salirse del lenguaje (los límites de mi lenguaje significan los límites de mi mundo, \$5.6) separa a priori el reino de lo que puede hablarse y el de aquello de lo cual no puede hablarse. El lenguaje queda atado aquí al "mundo natural".

De aquí que la estética ocupe, en esta primera etapa, el lugar de lo trascendental: queda fuera de los límites del lenguaje con sentido. Cuando queremos decir algo que no es factual -sino valorativo, como los juicios estéticos-, estamos entonces rebasando los límites de lo que puede ser dicho, los límites de la significatividad. La consecuencia entonces es que cualquier intento de proferir afirmaciones de valor cae en un sinsentido.

Por su parte, la otra concepción se presenta en muchos aspectos como radicalmente opuesta a la anterior, nos referimos a la que se desprende de las Investigaciones filosóficas, donde da cuenta del lenguaje desde la perspectiva de su uso, en conexión con las actividades humanas; y desde esta perspectiva, resulta imposible tener una única teoría del sentido (Tomasini Bassols 2012). Las nociones fundamentales que dan cuenta de este cambio son las de juego de lenguaje y forma de vida. Precisar esas categorías excede con mucho los límites del presente trabajo. Sin embargo, hay que decir que constituyen, ante todo, una embestida contra todo tipo de esencialismos. La expresión forma de vida es utilizada muy pocas veces por Wittgenstein (sólo dos en toda su obra), mientras que la de juego de lenguaje parece 
ser la central. El asunto es que el lenguaje queda mancomunado a las formas de vida de los hablantes. De ese modo, se elimina por completo la necesidad de suponer una estructura previa común entre lenguaje y rasgos del mundo (como en su primera filosofía). Por el contrario, el lenguaje se historiza y, si se nos permite la expresión, se humaniza. Dichos rasgos son precisamente los resaltados por Santiago Garmendia (2013) "una serie no homogénea de prácticas histórica y contingentemente regladas" (147). Lo que importa ahora es que las expresiones sean un movimiento genuino dentro de un juego del lenguaje. $Y$ esto es precisamente lo que debe tenerse en cuenta para comprender las expresiones más allá de su uso descriptivo. Pero para saber eso debemos fijarnos si esas expresiones tienen que ver con una práctica en la cual son significativas o si, por el contrario, se está haciendo girar una manivela en falso, como dice Wittgenstein en las Investigaciones Filosóficas. No otra cosa es su método de los juegos de lenguaje y las formas de vida. Otra manera de decirlo es que no hay términos que en sí mismos sean propios de un juego lingüístico: no hay términos que en sí mismos sean religiosos, ni científicos, ni artísticos, etcétera. Tomasini Bassols lo resume diciendo que "Es con la noción de movimiento en el juego de lenguaje que reemplazamos a la noción filosófica de proposición" (2012 11). Ya en el parágrafo §7 de las Investigaciones filosóficas Wittgenstein anuncia la fuerte ligazón entre lenguaje y praxis: "llamaré también juego de lenguaje al todo formado por el lenguaje y las acciones con las que está entretejido”, y más adelante en §23: “La expresión «juego de lenguaje» debe poner de relieve aquí que hablar el lenguaje forma parte de una actividad o de una forma de vida".

La metodología general que desarrolla en esta obra capital de su segundo periodo, la que ancla los juegos de lenguaje a las formas de vida y viceversa, implica pues que hay una conexión orgánica entre el lenguaje y la vida humana, una conexión interna: nuestra humanidad está conectada con nuestro lenguaje. Por ello quedan descartados los intentos de estudiar, por ejemplo, la "lógica" del lenguaje estético; es decir que una teoría formalista del lenguaje es enteramente inútil desde esta nueva óptica. En cambio, lo que se propone es una nueva metodología en la que se posa nuestra atención sobre la gramática, en sentido wittgensteiniano: como entretejido de usos y prácticas contextuales. Ahora el lenguaje está atado no ya al mundo natural, sino al "mundo social".

\section{¿Un arte sin criterios?}

Ahora bien, ante el panorama que nos presentan las prácticas artísticas contemporáneas la posibilidad de identificar criterios objetivos pareciera ser inalcanzable en comparación con otros momentos de la historia del arte; y ello 
debido a las características de este tipo de producción, en la cual nos encontramos con obras que no se inscriben dentro de un estilo, ni tampoco conforman un nuevo estilo. Ciertamente, la añadidura de 'contemporáneo' no designa un período, sino un modo de producir pluralista, que no respeta disciplinas, técnicas ni normas, que inventa y se renueva constantemente. Entonces, ¿en qué sentido podrá enunciarse un juicio que pueda ser considerado válido respecto de prácticas artísticas que precisamente parecen plantear la ausencia de criterios como su leitmotiv? $\mathrm{Y}$, de un modo más general y, con un cierto aire kantiano, podríamos preguntarnos ¿son posible los juicios estéticos ante este tipo de producciones?

En la concepción de la filosofía tradicional, la idea de verdad es reguladora, pues será la que determine el buen uso del lenguaje, esto es, cuándo hay adecuación entre una descripción (e.g., una proposición) y un hecho (un aspecto del mundo). En concordancia con esta concepción (episteme), la estética tradicional se asienta en la creencia de que existe una propiedad o grupo de propiedades comunes a todas las obras de arte y su tarea será entonces descubrir aquellas características distintivas que otorgan a un objeto el status de obra de arte (Bouveresse 1993). El problema es que no nos resulta nada fácil encontrar algo así como aires de familia entre obras artísticas a las que rotulamos como 'arte contemporáneo'. Problemas como el recién enunciado son vistos por Wittgenstein desde un terreno más amplio, el de su crítica a los lenguajes privados (y privilegiados) y la noción de regla. En su médula, la crítica se hace eco de la imposibilidad, en un lenguaje privado, de obtener parámetros de corrección (si yo soy mi propio juez lingüístico nunca puedo determinar cuándo cometo errores). La regla, pues, debe provenir de un contexto que exceda al propio sujeto; nunca aprendemos una proposición aislada, sino que nos sumergimos en un sistema de proposiciones. En el caso del arte, los criterios deben buscarse justamente en esas prácticas situadas, concretas y dinámicas de las que resulta la conexión lenguajevida social.

Según esto ya no podremos encontrar características suficientes y necesarias -criterios- que determinen que un objeto puede ser considerado una obra de arte, tampoco existe una lectura ideal de una obra ni, por tanto, criterios definitivos para juzgarlas. No por ello la identificación de un objeto cualquiera como obra de arte será una decisión arbitraria, sino que surgirá de su reconocimiento al interior de un juego del lenguaje y una forma de vida, o en términos del filósofo Arthur Danto de un mundo del arte ${ }^{2}$.

\footnotetext{
${ }^{2}$ Filósofo del arte de raigambre analítica que desarrolla el concepto de 'mundo del arte' para señalar que la identificación de objetos como obras de arte no dependen de propiedades o cualidades intrínsecas de aquellos objetos, sino de su inmersión en una época histórica determinada y de la "atmósfera conceptual" que
} 
Si tomamos la vía de Wittgenstein en su segundo período, donde abandona toda intención de generalizar (su método pasa a ser la ejemplificación de casos singulares, lo que él llama "casuística"), se evita el problema de encontrar una definición acabada de lo que sea el arte y dentro de éste los distintos movimientos, estilos y períodos que incluye, como ser el arte contemporáneo. La aplicación del método de los juegos del lenguaje y las formas de vida posibilita una aproximación al arte completamente nueva, nos permite dar cuenta de cómo se expande este concepto hasta incorporar producciones artísticas que se alejan completamente de la concepción de arte que se tenía hasta entonces; nos permite ver que la búsqueda de una definición de arte, la pregunta por el qué, por la esencia del arte, es una pregunta sin sentido. Gracias a este método los conceptos adquieren una nueva flexibilidad. Wittgenstein nos habla de la posibilidad de conceptos borrosos y nos dice en el parágrafo $\$ 71$ de las Investigaciones Filosóficas "¿pero es un concepto difuso en absoluto un concepto? ¿Es una fotografía difusa en absoluto una figura de una persona? Sí; ¿puede siempre reemplazarse con ventaja una figura difusa con una nítida? ¿No es a menudo la difusa la que realmente necesitamos?" Según esta concepción un concepto no necesita entonces de bordes nítidos o bien definidos, los conceptos son conceptos de semejanza de familia, y su sentido estará determinada por el uso que se haga de él en una determinada práctica.

Esta nueva condición de los conceptos nos lleva, entre otros, al problema de los indiscernibles planteada por Danto -reflexión provocada por las Brillo Box de Andy Warhol-: si un objeto cualquiera puede adquirir el status de obra de arte, qué es lo que lo distingue entonces de otro objeto igual que no lo sea, es decir, que no reciba esa distinción. A su vez, este problema de la indistinción nos devuelve, una vez más, al problema más general de los criterios, del que se desprende la siguiente pregunta: ¿cuándo un juicio puede considerarse válido en el campo del arte? Pues de lo que se trata no es tanto de buscar un criterio sempiterno cuanto de alcanzar un grado de comprensión del lugar que la obra ocupa como parte de una época en la cual adquiere sentido.

Una vez más: Wittgenstein no propone un sentido bajo el cual adquiere significado un período, un estilo, una corriente artística o una obra. Sino que ejemplifica, como consecuencia directa de su nueva filosofía del lenguaje, la manera en la que identificamos determinados objetos como obras de arte. Pero esa posibilidad viene dada por una praxis dentro de una comunidad, no por teorías canónicas ni manifiestos. Dicho en lenguaje wittgensteiniano, el asunto gira en torno a cuándo estamos haciendo un uso "indebido" de las reglas que rigen

predomina en ella, que es la que permite que identifiquemos determinados objetos y no otros como obras de arte. 
nuestro juego; cuándo hacemos que el lenguaje se vaya de vacaciones y cuándo efectivamente su uso está jugando un rol dentro de un entramado lingüístico y práctico. Los criterios, entonces, no pueden resultar tan caprichosos.

Esto está relacionado con que los significados no pueden, por lógica, depender de una esfera privada. La "vida" de los signos viene no de los misterios que ocurrirían en la caja negra de nuestra mente, sino de los usos públicos enraizados en las actividades teóricas y prácticas de los individuos concretos en contextos concretos, es decir, dependen del lugar que ocupen en un juego de lenguaje. Aprender las reglas de determinado juego estético es entonces involucrarse en una cultura: "describir completamente un paquete de reglas estéticas significa realmente describir la cultura de una época" (Wittgenstein 1992 72). En el caso de las producciones artísticas contemporáneas, por más variadas que sean sus manifestaciones, no pueden ser una expresión completamente aislada de las formas de vida en que surgen. Aunque proclamemos que el arte se vuelve sobre sí mismo, ese internalismo, como todo otro, tiene sus límites. Como bien dijo Wittgenstein en las Investigaciones \$580 “Un 'proceso interno' necesita criterios externos", lo cual no significa que el arte deba explicitar esos criterios. En parte, eso compete a teóricos y críticos de arte.

\section{Atender a las razones}

Ante este nuevo panorama en el mundo del arte, quien debe asumir el mayor desafío es el espectador. La ausencia de criterios claros y distintos exige de éste un rol más activo a la hora de enfrentarse y juzgar una obra, de aquí esta idea de que una buena solución debe ser cada vez inventada, no descubierta. Siguiendo esta línea, la lectura de una obra de arte contemporáneo implica un acto creativo a la vez que de raciocinio. Con esto nos referimos a que las nuevas experiencias que propone el arte contemporáneo ya no se prestan a una inmersión contemplativa a la que otrora invitaban las obras de arte tradicionales. Es así que la instancia de recepción de la obra se complejiza, el espectador ya no puede apelar o pretender una lectura "correcta" de la obra. Las nuevas exigencias que recaen sobre el espectador, el de ser un activo intérprete de una obra cuya lectura no se agota, provoca nuevas dificultades en la recepción de la obra, que van desde la incomprensión de la misma hasta los juicios apresurados. Por lo que muchos espectadores, antes que reconocer su ignorancia, zanjan la cuestión con sentencias como ser: "esto es cualquier cosa", "esto no es arte" o sencillamente "esto es una 
mierda"3, y ello se debe, en gran parte, a un anacronismo, es decir, al hecho de que aquellos juicios descansan en criterios propios de la estética tradicional.

El punto que interesa destacar es que el supuesto sinsentido de determinadas propuestas artísticas no viene dado tanto por una incorrección respecto a ningún tipo de parámetro, ya sea escrito o implícito, de un movimiento particular, sino por la incapacidad de entender la gramática profunda de las prácticas de un sistema. Justamente, sólo en la medida en que nos vemos inmersos en un juego de lenguaje -por ejemplo, el del arte contemporáneo-, es que somos capaces de hacer apreciaciones. Para hablar de la noción de apreciación debemos referirnos a la distinción que Wittgenstein establece entre razones y causas.

Dice Wittgenstein en los Cuadernos azul y marrón que “las palabras 'causa' y 'razón' tienen gramáticas totalmente diferentes" (1976 14-15). Una consecuencia significativa del giro social y pragmático de la filosofía de Wittgenstein es la mayor fecundidad de análisis que permite. Aquellas dimensiones como la religiosa, la estética, o la ética (las dimensiones valorativas) que otrora caían en el reino de lo indecible y por tanto sólo cabía iluminar en base a una más precisa delimitación del campo de lo decible, se vuelven ahora un campo fértil para la aplicación de su metodología basada en la descripción de casos, usos, ejemplificaciones, etc., que desnudan la gramática profunda de las expresiones lingüísticas en aras de una visión perspicua. Una de estas aplicaciones a que da lugar es la distinción entre los roles diferentes que pueden jugar expresiones como "por qué" en distintos juegos de lenguaje. Cuando se hace una pregunta de la forma "¿por qué?" podemos estar preguntando por causas o inquiriendo sobre razones. Cuando preguntamos acerca de una enfermedad, estamos esperando una respuesta de tipo causal (me resfrié porque tomé frío anoche). En cambio, cuando queremos comprender, buscamos razones. En el terreno del arte una explicación causal sería aquella que procurase rastrear lo que generó ciertos efectos en el espectador (por ejemplo, señalar que cierta tonalidad grisácea provoca una reacción melancólica). Es decir, aquella que busca los mecanismos que actuarían como resortes de la reacción, que sin ellos no se daría. En cambio, dar razones de algo es tratar de comprenderlo en una serie más amplia, ubicarlo en un entramado lingüístico y práctico de una cultura concreta. Por eso es que "Sólo en apariencia hacemos el mismo tipo de pregunta cuando buscamos las razones de una perplejidad estética y las de un empacho gástrico" (Wittgenstein 1992 79, el resaltado es nuestro).

\footnotetext{
${ }^{3}$ Con el arte moderno el comentario típico era "esto lo puede hacer mi hijo" (por ejemplo, frente a una obra de Jackson Pollock) y con el contemporáneo "esto es una mierda" es literalmente el primer juicio al que arriban muchos espectadores, pensemos las reacciones que provocaron y siguen provocando el mingitorio de Duchamp, o un ejemplo más literal y reciente "Mierda de artista" del artista conceptual Piero Manzoni.
} 
Ahora bien, si posamos la mirada en ese entramado de cultura, lenguaje y acción, pareciera ser que la obra de arte pasa a ocupar un lugar cuasi secundario. Toda manifestación cultural sería, de este modo, nada más que algún tipo de expresión de motivos que en principio no le son propios, sino extraídos de su época. En una palabra, la creatividad quedaría relegada a un segundo plano. Por el contrario, amén de su ubicación en un contexto histórico y social, las obras de arte no pierden su carácter creativo. El desafío que supone "leer" una obra de arte nos exige como espectadores establecer conexiones, interpretaciones, atribuciones de sentido, es decir, el objeto artístico nos impele a realizar una apreciación. La apreciación es lo que va más allá del nivel primitivo-orgánico de satisfacción, que a lo sumo generan juicios como "es hermoso", "es bello", y, en cambio, da cuenta de haberse familiarizado con una actividad y las reglas que la rigen.

Que las obras “arrastran” el mundo social del que forman parte queda muy bien ilustrado en la propuesta del filósofo francés Jacques Bouveresse. Este filósofo propone el concepto de claridad como clave del pensamiento wittgensteiniano y como una buena plataforma para comprender la transición entre sus dos filosofías. A este concepto lo vincula con dos nociones en el segundo Wittgenstein: el de la llamada "representación perspicua o sinóptica" y el de la percepción de figuras ambiguas. En relación con esta última, Wittgenstein habla del "fulgurar de un aspecto", que se vincula con el "ver cómo", esta percepción se produce "de golpe" $\mathrm{y}$, en este sentido, el concepto de claridad se vincula más con la acepción "resplandecer", "iluminarse", entonces esta claridad lo ilumina todo, pero por un momento (Bouveresse 1993).

Sin dudas esa iluminación que se produce cuando uno logra apreciar la obra de cierta forma está expresado en lo que Wittgenstein menciona como hacer un click: "se sugieren varias cosas y una de ellas hace click" (1992 86). Ese click es lo que me lleva, cuando leo un poema que antes no llamaba mi atención de una forma diferente a decir: "ah, ahora sé por qué hizo esto. Esto es grande" (1992 67). El criterio de que hicimos un "click", es que una explicación nos satisface, cuando decimos o pensamos "claro, ¡era esto!". Sentimos que comprendimos una obra cuando por ejemplo vislumbramos las razones que movieron al artista, o como dice Bouveresse "cuando leemos la obra de la manera indicada, una intención o un sentido nos aparecen, la obra nos habla, nos 'dice' algo, etc." (1993 38).

Pensemos como ejemplo la videoinstalación "Inefable" (2014) de la artista tucumana Evi Tartari. En esta pieza -según dice en la memoria conceptual-alude al concepto de desprendimiento, que implica el desplazamiento de una parte que pertenece a un todo. La artista quiere hablarnos de la acción de desprenderse, y ¿cómo hacerlo?, ¿cómo hablar de ella sin recurrir a definiciones de diccionario, descripciones o enumeraciones absurdas?, las cuales sólo lograrían alejarnos de lo 
que intenta decirnos. A lo largo de 30 minutos vemos como lentamente se va produciendo el desprendimiento de una raíz de la tierra, la raíz actúa como metáfora de lo familiar -de la genealogía- entonces ese desprendimiento puede leerse como su propio proceso de desprendimiento del lazo familiar. También ilumina esta pieza el resto de su producción, una serie de trabajos donde está presente la autorreferencialidad, el tema de la mujer, del cuerpo, los lazos familiares, etc. Esta obra, como tantas otras, exige reflexión y conocimiento, pide que uno aprecie sus razones.

Es decir que hay una suerte de dialéctica entre el objeto cultural y el sujeto que se para frente a él, entre "lo que éste le dice" y lo que él puede decir sobre esa obra. Los objetos en sí mismos no son significativos, pero lo que se interpreta es el objeto en aras de lo que Bouveresse llama búsqueda de significación: "El hombre es, podríamos decir, un animal perpetuamente en búsqueda de significación [...] el objeto no es un símbolo que debe ser interpretado; es cuando hemos aceptado una determinada interpretación cuando el objeto se convierte para nosotros en un símbolo" (1993 68).

\section{A modo de conclusión}

El arte contemporáneo obliga a poner sobre la mesa la discusión acerca de lo que puede ser considerado arte. ¿Cuáles son las contribuciones y los límites de incorporar análisis wittgensteinianos a la polémica? Vincular la discusión con nociones como las de juego de lenguaje permite no zanjarla por completo, pero tal vez nos da indicios para no tropezar con falsos problemas que muchas veces son los que obturan las arterias que conectan las obras de artes con su verdadero corazón: la vida de las personas. Los juegos son, a la vez, reglados y abiertos, dinámicos. Así, conectamos el lenguaje del arte con la incógnita intrínseca que acompaña lo lúdico, sin desmedro de la complejidad del "mundo del arte". No se elimina la dificultad propia de este juego de lenguaje infinitamente complejo como es el del arte (¿hace falta recordar que juego de lenguaje incluye en un todo a la vida artística, también a los "objetos" artísticos?), sólo se señala la infecundidad de buscar criterios inmóviles que solo fosilizan y obstaculizan la eclosión de la vida artística, tan rica y necesaria. No se solucionan todos los problemas; nos limitamos a señalar, entonces, la esterilidad de quien pretende reemplazar, arbitrariamente, una figura borrosa por una nítida.

La filosofía wittgensteiniana tiene la virtud de disolver lo que el vienés llamaba "pseudo-problemas" (buscar la esencia de la obra de arte), nacidos del embrujo que genera el mal uso del lenguaje. Queda por ver si no estamos 
atrapados en un nuevo embrujo, el de la primacía de la praxis. Pues la lección parece ser, como reza El Fausto, que en el principio no es el verbo, sino la acción.

\section{Bibliografía}

Bouveresse, Jacques. Wittgenstein y la estética. Valencia: Universitat de Valencia, 1993.

Garmendia, Santiago. Lenguaje y realidad en el Tractatus de Wittgenstein. Tucumán: EFFyL, 2013.

Danto, Arthur. La transfiguración del lugar común: una filosofía del arte. Barcelona: Paidós Ibérica, 2002.

Tomasini Bassols, Alejandro. "Wittgenstein: obra de arte y estética", Estéticas Contemporáneas, 6 (2014).

Tomasini Bassols, Alejandro. "La superioridad del método de los juegos del lenguaje y las formas de vida". Wittgenstein en Español III, ed. Alejandro Tomasini Bassols. México D. F: Universidad Veracruzana, 2012.

Vilar, Gerard. Las razones del arte. Madrid: Machado libros, 2005.

Wittgenstein, Ludwig. Lecciones y conversaciones sobre estética, psicología y creencia religiosa. Barcelona: Paidós Ibérica S.A., 1992.

Wittgenstein, Ludwig. Investigaciones Filosóficas. Barcelona: Altaya S.A., 1999.

Wittgenstein, Ludwig. Comentarios a la Rama Dorada de Frazer. México D.F.: Ed. UNAM, Ciudad Universitaria, 1985.

Wittgenstein, Ludwig. Los cuadernos azul y marrón. Madrid: Editorial Tecnos, 1976.

Wittgenstein, Ludwig. Tractatus Logico-Philosophicus. Madrid: Alianza, 1979. 